\title{
Algebraic Geometry for Computer-Aided Geometric Design
}

Thomas W. Sederberg

tom@cs.byu.edu

Ronald N. Goldman

Follow this and additional works at: https://scholarsarchive.byu.edu/facpub

Part of the Computer Sciences Commons

\section{Original Publication Citation}

Sederberg, T. W., and R. N. Goldman. "Algebraic Geometry for Computer-Aided Geometric Design." Computer Graphics and Applications, IEEE 6.6 (1986): 52-9.

\section{BYU ScholarsArchive Citation}

Sederberg, Thomas W. and Goldman, Ronald N., "Algebraic Geometry for Computer-Aided Geometric Design" (1986). Faculty Publications. 753.

https://scholarsarchive.byu.edu/facpub/753

This Peer-Reviewed Article is brought to you for free and open access by BYU ScholarsArchive. It has been accepted for inclusion in Faculty Publications by an authorized administrator of BYU ScholarsArchive. For more information, please contact ellen_amatangelo@byu.edu. 


\title{
Algebraic Geometry for Computer-Aided Geometric Design \\ Thomas W. Sederberg \\ Brigham Young University
}

\author{
Ronald N. Goldman \\ Control Data Corporation
}

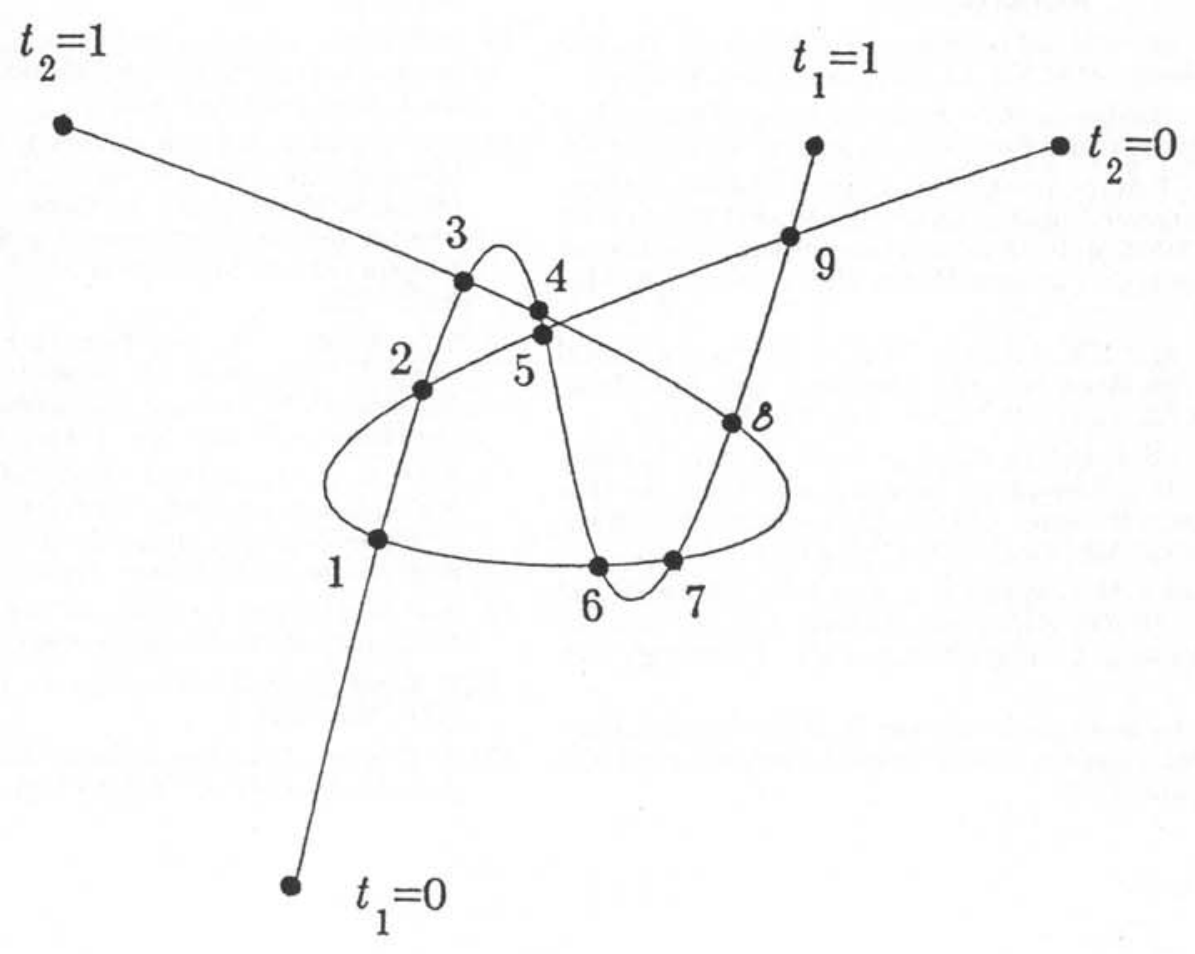

Classical algebraic geometry has been virtually ignored in computer-aided geometric design. However, because it deals strictly with algorithms, it is really more suited to this field than is modern algebraic geometry, which introduces abstractions far removed from the algorithmic nature of computer-aided design.

This tutorial examines resultants, curve implicitization, curve inversion, and curve intersection. Discussion follows a series of examples simple enough for those with only a modest algebra background to follow.

\section{C} omputer-aided geometric design and graphics has drawn heavily from differential geometry and vector geometry, but is only beginning to access the tools of classical algebraic geometry. We underscore the word classical because much modern algebraic geometry deals with abstractions that are far removed from the algorithmic nature of computer-aided geometric design and graphics, whereas classical mathematicians-those of 50 to 150 years ago-tended to write in less abstract terms. In this article, we discuss three problems, which were first

discussed a century ago but which have lain dormant for decades. The discussion is intended for a general technical audience and presupposes only a modest background in algebra. The concepts are motivated by simple examples, and no rigorous proofs are included. Other references provide the interested reader with lucid expositions of the underlying theory ${ }^{1-3}$ and advanced and specialized treatments. ${ }^{4,5}$ We will discuss the following three problems:

1. Implicitization problem. Given a planar curve defined parametrically as

$$
x=\frac{x(t)}{w(t)}, y=\frac{y(t)}{w(t)}
$$

where $x(t), y(t)$, and $w(t)$ are polynomials, find an implicit equation $f(x, y)=0$ that defines the same curve.

2. Inversion problem. Given the $x, y$ coordinates of a point that lies on a parametric curve

$$
x=\frac{x(t)}{w(t)}, \quad y=\frac{y(t)}{w(t)}
$$

find the parameter value $t$ that corresponds to this point. 
3. Intersection problem. Compute the points of intersection of two parametric curves using the implicitization and inversion techniques.

We discuss implicitization and inversion together and then apply them to the intersection problem. Several works in computer algebra deal with these topics, ${ }^{6,7}$ and there are several works on the implicitization of curves ${ }^{8,9}$ and the implicitization of surfaces. ${ }^{10,11}$ There are also other applications of algebraic geometry to problems in computer-aided geometric design. ${ }^{12,13}$

\section{Curve representation}

Before we discuss these problems, let us look at curve representation in general. Basically, a planar curve can be defined in two ways: parametrically and implicitly. The parametric equation of a plane curve takes the form

$$
x=\frac{x(t)}{w(t)} \quad y=\frac{y(t)}{w(t)}
$$

and the implicit equation of a curve is of the form

$$
f(x, y)=0
$$

In these notes, we restrict ourselves to the case where $x(t), y(t), w(t)$, and $f(x, y)$ are polynomials.

Obviously, the parametric equation of a curve has the advantage of being able to quickly compute the $x, y$ coordinates of several points on the curve for plotting. Also, it is simple to define a curve segment by restricting the parameter $t$ to a finite range, for example $0 \leq t \leq 1$. On the other hand, the implicit equation of a curve enables us to easily determine whether a given point lies on the curve, or if not, which side of the curve it lies on.

Given that we have two different equations for curves, can we convert between representations for a given curve? The answer is yes; it is always possible to find an implicit equation of a parametric curve, but a parametric equation can generally be found only for implicit curves of degree two or one. Later in this article, we will discuss how to convert between representations using an important algebraic tool called the resultant. For fun, we also show how someone might tackle the implicitization problem before learning about resultants.

\section{Brute-force implicitization}

Consider this simple example of parametric-toimplicit conversion. Given a line

$$
x=t+2 \quad y=3 t+1
$$

we can easily find an implicit equation that identically represents this line by solving for $t$ as a function of $x$

$$
t=x-2
$$

and substituting into the equation for $y$

$$
y=3(x-2)+1
$$

or $3 x-y-5=0$. Note that this implicit equation defines precisely the same curve as does the parametric equation. We can also identify two inversion equations (for finding the parameter value of a point on the line): $t=x-2$ or $t=(y-1) / 3$.

This approach to implicitization also works for degreetwo parametric curves. Consider the parabola

$$
x=t^{2}+1 \quad y=t^{2}+2 t-2
$$

Again, we can solve for $t$ as a function of $x$ :

$$
t= \pm \sqrt{x-1}
$$

and substitute into the equation for $y$ :

$$
y=(\sqrt{x-1})^{2} \pm 2 \sqrt{x-1}-2
$$

We can isolate the radical and square both sides

$$
(y-(x-1)+2)^{2}=( \pm 2 \sqrt{x-1})^{2}
$$

to yield

$$
x^{2}-2 x y+y^{2}-10 x+6 y+13=0
$$

which is the desired implicit equation. Again this implicit equation defines exactly the same curve as does the parametric equation.

We run into trouble, however, if we try to apply this implicitization technique to curves of degree higher than two. The critical step is expressing $t$ as a function of $x$. For cubic and quartic equations, it can be done, but the resulting expression is hopelessly complex. For curves of degree greater than four, it cannot be done at all.

Further, we cannot obtain an inversion equation for this parabola the way we did for the straight line. For example, suppose we want to find the parameter of the point $(5,-2)$, which we know lies on the curve. The bruteforce approach is to find the values of $t$ that satisfy the equation

$$
x=5=t^{2}+1
$$

and then to compare them with the values of $t$ that satisfy the equation

$$
y=-2=t^{2}+2 t-2
$$

In the first case $t=-2$ or 2 , and in the second case $t=-2$ or 0 . The value of $t$ that satisfies both equations is -2 , which must therefore be the parameter value of the point $(5,-2)$.

\section{Resultants}

Thus far our attempt at implicitization and inversion has been unsuccessful. An elegant, general solution to the problem is provided by what are called resultants. $\mathrm{Re}$ sultants address the question of whether two polynomials have a common root. Consider the two polynomials

$$
f(t)=\sum_{i=0}^{n} a_{i} t^{i} \quad g(t)=\sum_{i=0}^{n} b_{i} t^{i}
$$

The resultant of $f(t)$ and $g(t)$, written $R(f, g)$, is an expression in terms of the coefficients $a_{i}$ and $b_{i}$ such that a common root of $f(t)$ and $g(t)$ exists if and only if $R(f, g)$ $=0$. 
We illustrate by finding the resultant of two cubic polynomials

$$
\begin{aligned}
& f(t)=a_{3} t^{3}+a_{2} t^{2}+a_{1} t+a_{0} \\
& g(t)=b_{3} t^{3}+b_{2} t^{2}+b_{1} t+b_{0}
\end{aligned}
$$

In other words, we want to determine whether a value $\alpha$ exists such that $f(\alpha)=g(\alpha)=0$-without actually finding and comparing all roots of both polynomials. We begin by forming three auxiliary polynomials $h_{1}(t), h_{2}(t)$, and $h_{3}(t)$ as follows:

$$
\begin{aligned}
& h_{1}(t)=a_{3} g(t)-b_{3} f(t) \\
& =\left(a_{3} b_{2}\right) t^{2}+\left(a_{3} b_{1}\right) t+\left(a_{3} b_{0}\right) \\
& \text { where }(a, b,) \equiv(a, b,-a, b i) \text { and } \\
& h_{2}(t)=\left(a_{3} t+a_{2}\right) g(t)-\left(b_{3} t+b_{2}\right) f(t) \\
& =\left(a_{3} b_{1}\right) t^{2}+\left[\left(a_{3} b_{0}\right)+\left(a_{2} b_{1}\right)\right] t+\left(a_{2} b_{0}\right) \\
& h_{3}(t)=\left(a_{3} t^{2}+a_{2} t+a_{1}\right) g(t)-\left(b_{3} t^{2}+b_{2} t+b_{1}\right) f(t) \\
& =\left(a_{3} b_{0}\right) t^{2}+\left(a_{2} b_{0}\right) t+\left(a_{1} b_{0}\right)
\end{aligned}
$$

Note that if a value $\alpha$ exists such that $f(\alpha)=g(\alpha)=0$, then $h_{1}(\alpha)=h_{2}(\alpha)=h_{3}(\alpha)=0$. We can therefore say that $f(t)$ and $g(t)$ have a common root if and only if the set of equations

$$
\left[\begin{array}{ccc}
\left(a_{3} b_{2}\right) & \left(a_{3} b_{1}\right) & \left(a_{3} b_{0}\right) \\
\left(a_{3} b_{1}\right) & \left(a_{3} b_{0}\right)+\left(a_{2} b_{1}\right) & \left(a_{2} b_{0}\right) \\
\left(a_{3} b_{0}\right) & \left(a_{2} b_{0}\right) & \left(a_{1} b_{0}\right)
\end{array}\right]\left\{\begin{array}{l}
t^{2} \\
t \\
1
\end{array}\right\}=0
$$

has a solution. (Actually, we have shown only that this condition is necessary; the proof that it is also sufficient is found elsewhere. ${ }^{14}$ ) However, we know from linear algebra that this set of homogeneous linear equations can have a solution if and only if



and therefore,

$$
R(f, g)=\left\{\begin{array}{ccc}
\left(a_{3} b_{2}\right) & \left(a_{3} b_{1}\right) & \left(a_{3} b_{0}\right) \\
\left(a_{3} b_{1}\right) & \left(a_{3} b_{0}\right)+\left(a_{2} b_{1}\right) & \left(a_{2} b_{0}\right) \\
\left(a_{3} b_{0}\right) & \left(a_{2} b_{0}\right) & \left(a_{1} b_{0}\right)
\end{array} \mid\right.
$$

The same approach can be used to construct the resultant of polynomials of any degree. (Other sources present a more detailed algorithm for forming the elements of the determinant. ${ }^{14}$ )

\section{Applying the concept}

Let's now try the resultant approach on a couple of examples. First, let $f(t)=t^{3}-2 t^{2}+3 t+1$ and $g(t)=2 t^{3}+$ $3 t^{2}-t+4$. For this case,

$$
R(f, g)=\left|\begin{array}{ccc}
7 & -7 & 2 \\
-7 & -5 & -11 \\
2 & -11 & 13
\end{array}\right|=-1611
$$

We aren't interested so much in the actual numerical value of the resultant, just whether or not it equals zero. In this case, $R(f, g)=-1611 \neq 0$, so we conclude that $f(t)$ and $g(t)$ do not have a common root.

Let us next consider the pair of polynomials $f(t)=t^{3}-$ $t^{2}-11 t-4$ and $g(t)=2 t^{3}-7 t^{2}-5 t+4$. In this case

$$
R(f, g)=\left|\begin{array}{ccc}
-5 & 17 & 12 \\
17 & -60 & -32 \\
12 & -32 & -64
\end{array}\right|=0
$$

since $R(f, g)=0, f(t)$ and $g(t)$ do have a common root. Note that the resultant simply determines the existence or nonexistence of a common root but does not directly reveal the value of a common root, if one exists. In fact, if the resultant is zero, there may actually be several common roots.

\section{Determining the common root}

We present two basic approaches to finding the common root of two polynomials: (1) solving a set of linear equations and (2) using Euclid's algorithm.

\section{Linear equation approach}

Our intuitive development of the resultant of two cubic polynomials led us to a set of three linear equations in three unknowns: $t^{2}, t$, and 1 . In general, we could create the resultant of two degree- $n$ polynomials

$$
f(t)=a_{n} t^{n}+a_{n-1} t^{n-1}+\cdots+a_{1} t+a_{0}
$$

and

$$
g(t)=b_{n} t^{n}+b_{n-1} t^{n-1}+\cdots+b_{1} t+b_{0}
$$

as the determinant of the coefficient matrix of $n$ homogeneous linear equations:

$$
\left[\begin{array}{cccc}
\left(a_{n} b_{n-1}\right) & \cdot & \cdot & \left(a_{n} b_{0}\right) \\
\cdot & \cdot & \cdot & \cdot \\
\cdot & \cdot & \cdot & \cdot \\
\cdot & \cdot & \cdot & \cdot \\
\left(a_{n} b_{0}\right) & \cdot & \cdot & \left(a_{1} b_{0}\right)
\end{array}\right]\left\{\begin{array}{c}
t^{n-1} \\
t^{n-2} \\
\cdot \\
\cdot \\
t \\
1
\end{array}\right\}=0
$$

It may be a bit confusing at first to view this as a set of homogeneous linear equations, since all the unknowns are powers of $t$. Let us temporarily rename our unknowns:

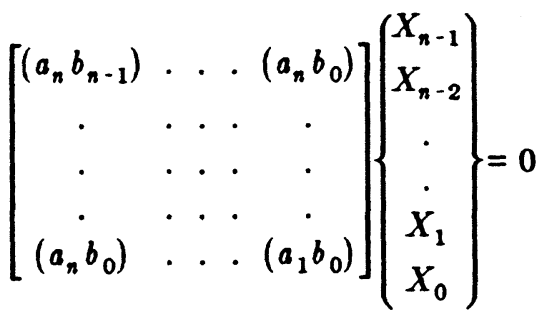


where $X_{i}=t^{i}$. After solving for $X_{i}$, the common root of $f(t)$ and $g(t)$ can be obtained as $t=X_{i+1} / X_{i}$.

\section{Cramer's rule}

There are several well-known methods of solving for $X_{i}$. One way is to apply Cramer's rule. A nontrivial solution exists (that is, a solution other than $X_{0}=X_{1}=\ldots$. $=X_{n-1}=0$ ) only if the determinant of the matrix is zero. But, that implies that the $n$ equations are linearly dependent, and we can discard one of them without losing any information. We discard the last equation, and then solve the remaining $n-1$ homogeneous equations in $n$ homogeneous unknowns using Cramer's rule. It turns out that occasionally we run into trouble if we discard an equation other than the last one. ${ }^{14}$ We illustrate Cramer's rule for the case $f(t)=t^{3}-t^{2}-11 t-4$ and $g(t)=2 t^{3}-7 t^{2}$ $-5 t+4$. Recall that for this pair we have established that $R(f, g)=0$. We have the set of equations

$$
\left[\begin{array}{ccc}
-5 & 17 & 12 \\
17 & -60 & -32 \\
12 & -32 & -64
\end{array}\right]\left\{\begin{array}{l}
X_{2} \\
X_{1} \\
X_{0}
\end{array}\right\}=0
$$

Discarding the last equation, we obtain

$$
\left[\begin{array}{ccc}
-5 & 17 & 12 \\
17 & -60 & -32
\end{array}\right]\left\{\begin{array}{l}
X_{2} \\
X_{1} \\
X_{0}
\end{array}\right\}=0
$$

from which we find the common root using Cramer's rule:

$$
t=\frac{X_{1}}{X_{0}}=-\frac{\left|\begin{array}{cc}
-5 & 12 \\
17 & -32
\end{array}\right|}{\left|\begin{array}{cc}
-5 & 17 \\
17 & -60
\end{array}\right|}=4
$$

\section{Gauss elimination}

A numerically superior algorithm for solving this set of equations is to perform Gauss elimination. Two other advantages of Gauss elimination are that (1) it can be used to ascertain whether the determinant is zero to begin with and (2) it reveals how many common roots there are. We will illustrate this approach with three examples, using integer-preserving Gauss elimination. We choose integer-preserving Gauss elimination because the lower right determinant element of the upper triangular matrix then becomes the value of the matrix.

Example 1: Our first example is one we considered earlier: $f(t)=t^{3}-2 t^{2}+3 t+1$ and $g(t)=2 t^{3}+3 t^{2}-t+4$. We set up the following set of linear equations and triangularize the matrix using integer-preserving Gauss elimination:

$$
\left[\begin{array}{ccc}
7 & -7 & 2 \\
-7 & -5 & -11 \\
2 & -11 & 13
\end{array}\right]\left\{\begin{array}{l}
X_{2} \\
X_{1} \\
X_{0}
\end{array}\right\}=\left[\begin{array}{ccc}
7 & -7 & 2 \\
0 & -84 & -63 \\
0 & 0 & -1611
\end{array}\right]\left\{\begin{array}{l}
X_{2} \\
X_{1} \\
X_{0}
\end{array}\right\}=0
$$

We observe that the only solution to this set of equations is $X_{2}=X_{1}=X_{0}=0$, and conclude that $f(t)$ and $g(t)$ do not have a common root. Note that the lower right element
-1611 is the determinant of the matrix, or the resultant.

Example 2: We next examine the pair of polynomials $f(t)=t^{3}-t^{2}-11 t-4$ and $g(t)=2 t^{3}-7 t^{2}-5 t+4$. In this case we have

$$
\left[\begin{array}{ccc}
-5 & 17 & 12 \\
17 & -60 & -32 \\
12 & -32 & -64
\end{array}\right]\left\{\begin{array}{l}
X_{2} \\
X_{1} \\
X_{0}
\end{array}\right\}=\left[\begin{array}{ccc}
-5 & 17 & 12 \\
0 & 11 & -44 \\
0 & 0 & 0
\end{array}\right]\left\{\begin{array}{l}
X_{2} \\
X_{1} \\
X_{0}
\end{array}\right\}=0
$$

Again, the lower right element is the value of the determinant, which verifies that the resultant is zero. It is now simple to compute the solution: $X_{1}=4 X_{0}, X_{2}=4 X_{1}$. Since $X_{i}=t^{i}$, the common root is $t=4$.

Example 3: For our final example we analyze the polynomials $f(t)=t^{3}-6 t^{2}+11 t-6$ and $g(t)=t^{3}-7 t^{2}+$ $14 t-8$. Our linear equations now are

$$
\left[\begin{array}{ccc}
-1 & 3 & -2 \\
3 & -9 & 6 \\
-2 & 6 & -4
\end{array}\right]\left\{\begin{array}{l}
X_{2} \\
X_{1} \\
X_{0}
\end{array}\right\}=\left[\begin{array}{ccc}
-1 & 3 & -2 \\
0 & 0 & 0 \\
0 & 0 & 0
\end{array}\right]\left\{\begin{array}{l}
X_{2} \\
X_{1} \\
X_{0}
\end{array}\right\}=0
$$

In this case, not only is the resultant zero, but the matrix is rank 1. In other words, there are two common roots, and they can be found as the solutions to the quadratic equation $-t^{2}+3 t-2=0$, which are $t=1$ and $t=2$. Consequently, $-t^{2}+3 t-2$ is the greatest common divisor, or GCD, of $f(t)$ and $g(t)$.

\section{Euclid's GCD algorithm}

An alternative approach to finding the common $\operatorname{root}(\mathrm{s})$ of two polynomials is to use Euclid's algorithm. There is a close connection between this algorithm and resultants. In fact, Euclid's algorithm does everything that resultants do. It is an ancient method of finding the GCD of two integers or two polynomials. Our presentation of Euclid's algorithm consists of a series of examples; Kurosh ${ }^{1}$ gives a clear proof. The algorithm works beautifully in exact integer arithmetic, but we have experienced numerical instability in floating point.

\section{Integers}

We illustrate first on a pair of integers: 42 and 30. First we assign the larger to be the numerator and the other to be the denominator:

$$
\text { Step 1: } \frac{42}{30}=1 \text { remainder } 12 \text {. }
$$

We now take the remainder of the first step and divide it into the denominator of the first step:

$$
\text { Step 2: } \frac{30}{12}=2 \text { remainder } 6 \text {. }
$$

We continue dividing the remainder of the preceding step into the denominator of the preceding step, until we obtain a zero remainder. This happens to occur in the third step for this problem:

Step 3: $\frac{12}{6}=2$ exactly.

According to Euclid's algorithm, the second-to-last remainder is the GCD. In this case, the second-to-last remainder is 6 , which is clearly the largest integer that evenly divides 30 and 42 . 


\section{Polynomials}

Example 1: We illustrate how Euclid's algorithm works for polynomials by using the same three examples we used in the previous section. For the polynomials $f(t)=t^{3}$ $-2 t^{2}+3 t+1$ and $g(t)=2 t^{3}+3 t^{2}-t+4$, we have

Step 1: $\frac{2 t^{3}+3 t^{2}-t+4}{t^{3}-2 t^{2}+3 t+1}=2$ remainder $7 t^{2}-7 t+2$

Step 2: $\frac{t^{3}-2 t^{2}+3 t+1}{7 t^{2}-7 t+2}=\frac{t-1}{7}$ remainder $\frac{12 t+9}{7}$

Step 3: $\frac{7 t^{2}-7 t+2}{(12 t+9) / 7}=\frac{196 t-343}{48}$ remainder $\frac{1253}{16}$

Step 4: $\frac{(12 t+9) / 7}{1253 / 16}=\frac{192 t}{8771}+\frac{144}{8771}$ remainder 0

In this case the GCD is $1253 / 16$, which is merely a constant, and so $f(t)$ and $g(t)$ do not have a common root.

Example 2: We next analyze the polynomials $f(t)=t^{3}$ $t^{2}-11 t-4$ and $g(t)=2 t^{3}-7 t^{2}-5 t+4:$

Step 1: $\frac{2 t^{3}-7 t^{2}-5 t+4}{t^{3}-t^{2}-11 t-4}=2$ remainder $-5 t^{2}+17 t+12$

Step 2: $\frac{t^{3}-t^{2}-11 t-4}{-5 t^{2}+17 t+12}=-5 t-\frac{12}{25}$ remainder $\frac{-11 t+44}{25}$

Step 3: $\frac{-5 t^{2}+17 t+12}{(-11 t+44) / 25}=125 t+\frac{75}{11}$ remainder 0

In this case, the GCD is $\frac{-\mathbf{1 1} t+\mathbf{4 4}}{25}$, and the common root is $t=4$.

Example 3: Finally, we consider $f(t)=t^{3}-6 t^{2}+11 t-6$ and $g(t)=t^{3}-7 t^{2}+14 t-8$ :

Step 1: $\frac{t^{3}-6 t^{2}+11 t-6}{t^{3}-7 t^{2}+14 t-8}=1$ remainder $t^{2}-3 t+2$

Step 2: $\frac{t^{3}-7 t^{2}+14 t-8}{t^{2}-3 t+2}=t-4$ remainder 0

The GCD is $t^{2}-3 t+2$, and the common roots are the roots of the equation $t^{2}-3 t+2=0$, which are $t=1$ and $t=2$.

\section{Implicitization and inversion}

We have just discussed resultants, a powerful tool for determining whether two polynomials have a common root. We want to apply that tool to converting the parametric equation of a curve given by

$$
x=\frac{x(t)}{w(t)}, y=\frac{y(t)}{w(t)}
$$

into an implicit equation of the form $f(x, y)=0$. We proceed by forming two auxiliary polynomials:

$$
p(x, t)=w(t) x-x(t) \quad q(y, t)=w(t) y-y(t)
$$

Note that $p(x, t)=q(y, t)=0$ only for values of $x, y$, and $t$, that satisfy the relationships

$$
x=\frac{x(t)}{w(t)} \text { and } y=\frac{y(t)}{w(t)} .
$$

View $p(x, t)$ as a polynomial in $t$ whose coefficients are linear in $x$, and view $q(y, t)$ as a polynomial in $t$ whose coefficients are linear in $y$. If

$$
x(t)=\sum_{i=0}^{n} a_{i} t^{i}, \quad y(t)=\sum_{i=0}^{n} b_{i} t^{i}, \text { and } w(t)=\sum_{i=0}^{n} d_{i} t^{i}
$$

then

$$
\begin{aligned}
p(x, t)= & \left(d_{n} x-a_{n}\right) t^{n}+\left(d_{n-1} x-a_{n-1}\right) t^{n-1}+\cdots \\
& +\left(d_{1} x-a_{1}\right) t+\left(d_{0} x-a_{0}\right) \\
q(y, t)= & \left(d_{n} y-b_{n}\right) t^{n}+\left(d_{n-1} y-b_{n-1}\right) t^{n-1}+\cdots \\
& +\left(d_{1} y-b_{1}\right) t+\left(d_{0} y-b_{0}\right)
\end{aligned}
$$

If we now compute the resultant of $p(x, t)$ and $q(y, t)$, we do not arrive at a numerical value, but rather a polynomial in $x$ and $y$, which we call $f(x, y)$. Clearly any $x, y$ pair for which $f(x, y)=0$ causes the resultant of $p$ and $q$ to be zero. But if the resultant is zero, then we know that a value of $t$ exists for which $p(x, t)=q(y, t)=0$. In other words, all $x, y$ values for which $f(x, y)=0$ lie on the parametric curve, and therefore $f(x, y)=0$ is the implicit equation of that curve. The following examples clarify.

Example 1: In the first example we apply this technique to the parabola we implicitized earlier using a brute-force method:

$$
x=t^{2}+1 \quad y=t^{2}+2 t-2
$$

We begin by forming $p(x, t)=-t^{2}+(x-1)$ and $q(y, t)=$ $-t^{2}-2 t+(y+2)$. The resultant of two quadratic polynomials $a_{2} t^{2}+a_{1} t+a_{0}$ and $b_{2} t^{2}+b_{1} t+b_{0}$ is

$$
\left|\begin{array}{ll}
\left(a_{2} b_{1}\right) & \left(a_{2} b_{0}\right) \\
\left(a_{2} b_{0}\right) & \left(a_{1} b_{0}\right)
\end{array}\right|
$$

and so the resultant of $p(x, t)$ and $q(y, t)$ is

$$
\begin{aligned}
& R(p, q)=\left|\begin{array}{cc}
2 & x-y-3 \\
x-y-3 & 2 x-2
\end{array}\right| \\
& =-x^{2}+2 x y-y^{2}+10 x-6 y-13
\end{aligned}
$$


which is the implicit equation we had obtained earlier.

We can write an inversion equation for this curvesomething that eluded us in our ad hoc approach:

$$
\left[\begin{array}{cc}
2 & x-y-3 \\
x-y-3 & 2 x-2
\end{array}\right]\left\{\begin{array}{l}
t \\
1
\end{array}\right\}=0
$$

from which

$$
t=\frac{-x+y+3}{2} \text { or } t=\frac{-2 x+2}{x-y-3}
$$

Example 2: We next implicitize the cubic curve (Figure 1) for which

$$
\begin{aligned}
& x=\frac{2 t^{3}-18 t^{2}+18 t+4}{-3 t^{2}+3 t+1} \\
& y=\frac{39 t^{3}-69 t^{2}+33 t+1}{-3 t^{2}+3 t+1}
\end{aligned}
$$

We begin by forming $p(x, t)$ and $q(y, t)$ :

$$
\begin{aligned}
& p(x, t)=-2 t^{3}+(-3 x+18) t^{2}+(3 x-18) t+(x-4) \\
& q(y, t)=-39 t^{3}+(-3 y+69) t^{2}+(3 y-33) t+(y-1)
\end{aligned}
$$

Recalling from our discussion on resultants that the resultant of two cubic polynomials $a_{3} t^{3}+a_{2} t^{2}+a_{1} t+a_{0}$ and $a_{3} t^{3}+a_{2} t^{2}+a_{1} t+a_{0}$ is

$$
\left\{\begin{array}{ccc}
\left(a_{3} b_{2}\right) & \left(a_{3} b_{1}\right) & \left(a_{3} b_{0}\right) \\
\left(a_{3} b_{1}\right) & \left(a_{3} b_{0}\right)+\left(a_{2} b_{1}\right) & \left(a_{2} b_{0}\right) \\
\left(a_{3} b_{0}\right) & \left(a_{2} b_{0}\right) & \left(a_{1} b_{0}\right)
\end{array} \mid\right.
$$

we have

$$
\begin{gathered}
R(p, q)=f(x, y)= \\
\left|\begin{array}{c}
-117 x+69 y+564117 x-6 y-636-39 x-2 y-154 \\
117 x-6 y-636-69 x-2 y+494-66 x+6 y+258 \\
39 x-2 y-154-66 x-2 y+25830 x-6 y-114
\end{array}\right|
\end{gathered}
$$

We can expand the determinant to get

$$
\begin{aligned}
f(x, y)= & -156195 x^{3}+60426 x^{2} y-7056 x y^{2}+224 y^{3} \\
& +2188998 x^{2}-562500 x y+33168 y^{2} \\
& -10175796 x+1322088 y+15631624
\end{aligned}
$$

We can obtain an inversion equation using Cramer's rule:

$$
t=\frac{t^{2}}{t}=\frac{\left|\begin{array}{cc}
117 x-6 y-636 & 39 x-2 y-154 \\
-69 x-2 y+494 & -66 x+6 y+258
\end{array}\right|}{\left|\begin{array}{cc}
-117 x+69 y+564 & 39 x-2 y-154 \\
117 x-6 y-636 & -66 x+6 y+258
\end{array}\right|}
$$

Alternatively, we could use Gauss elimination to compute the parameter of a point on the curve. Cramer's rule is appealing because it actually generates an equation.

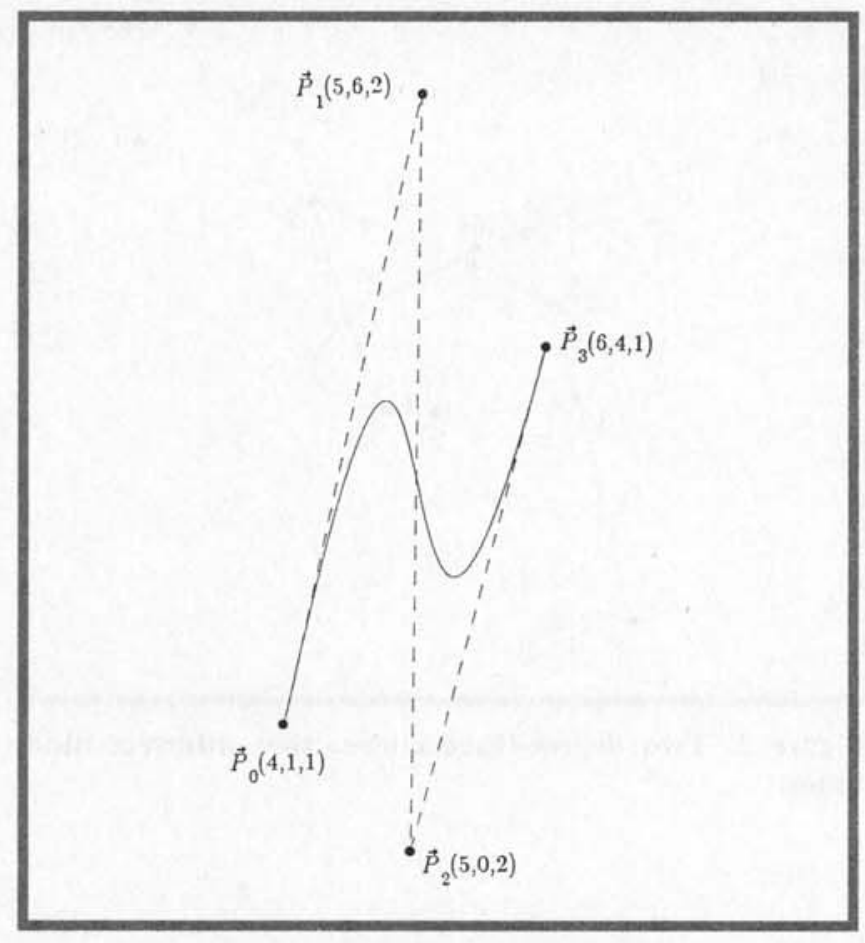

Figure 1. Cubic curve in implicitization Example 2. The points $\boldsymbol{P}_{i}$ are the Bezier control points of the curve. The third coordinate is a weight.

We have intentionally carried out all computations in exact integer arithmetic to emphasize the rational, noniterative nature of implicitization and inversion. Since the coefficients of the implicit equation are obtained from the coefficients of the parametric equations using only multiplication, addition, and subtraction, we can obtain an implicit equation that precisely defines the same point set defined by the parametric equations.

\section{Curve-curve intersections}

Given one curve defined by the implicit equation $f(x, y)$ $=0$ and a second curve defined by the parametric equations $x=x(t), y=y(t)$, we replace all occurrences of $x$ in the implicit equation by $x(t)$, and replace all occurrences of $y$ in the implicit equation by $y(t)$. These substitutions create a polynomial $f(x(t), y(t))=g(t)$ whose roots are the parameter values of the points of intersection. Of course, if we start off with two parametric curves, we can first implicitize one of them.

We illustrate this process ${ }^{15}$ by intersecting the curve

$$
\begin{aligned}
& x=\frac{2 t_{1}^{3}-18 t_{1}^{2}+18 t_{1}+4}{-3 t_{1}^{2}+3 t_{1}+1} \\
& y=\frac{39 t_{1}^{3}-69 t_{1}^{2}+33 t_{1}+1}{-3 t_{1}^{2}+3 t_{1}+1}
\end{aligned}
$$

with the curve 


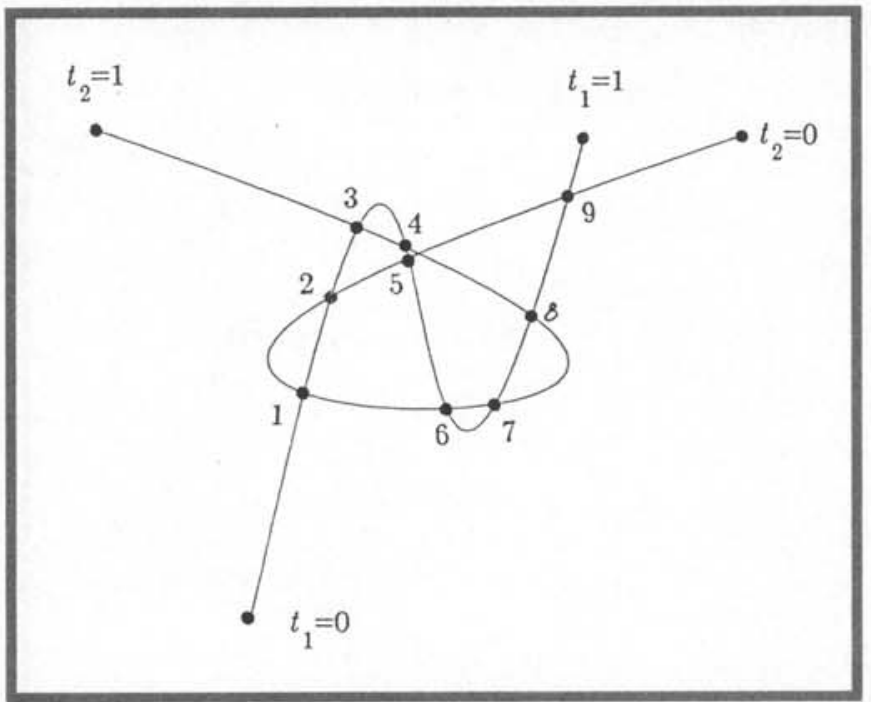

Figure 2. Two degree-three curves that intersect nine times.

$$
\begin{gathered}
x=\frac{-52 t_{2}^{3}+63 t_{2}^{2}-15 t_{2}+7}{-37 t_{2}^{2}+3 t_{2}+1} \\
y=\frac{4}{-37 t_{2}^{2}+3 t_{2}+1}
\end{gathered}
$$

The two curves intersect nine times (Figure 2), which is the most that two cubic curves can intersect."

We have already implicitized the first curve (in our discussion on implicitization and inversion), so our intersection problem requires us to make the substitutions

$$
\begin{aligned}
& x=\frac{-52 t_{2}^{8}+63 t_{2}^{2}-15 t_{2}+7}{-37 t_{2}^{2}+3 t_{2}+1} \\
& y=\frac{4}{-37 t_{2}^{2}+3 t_{2}+1}
\end{aligned}
$$

into the implicit equation of curve 1:

$$
\begin{aligned}
f(x, y)= & -156195 x^{3}+60426 x^{2} y-7056 x y^{2}+224 y^{3} \\
& +2188998 x^{2}-562500 x y+33168 y^{2} \\
& -10175796 x+1322088 y+15631624
\end{aligned}
$$

\footnotetext{
-Bezout's theorem states that two curves of degree $m$ and $n$, respectively, intersect in $m n$ points, if we include complex points, points at infinity, and multiple intersections.
}

After multiplying through by $\left(-37 t_{2}^{2}+3 t_{2}+1\right)^{3}$ we arrive at the intersection equation:

$$
\begin{aligned}
& 984100 t_{2}^{0}-458200 t_{2}^{8}+8868537 t_{2}^{7}-9420593 t_{2}^{6} \\
& +5949408 t_{2}^{5}-2282850 t_{2}^{4}+522890 t_{2}^{3}-67572 t_{2}^{2} \\
& +4401 t_{2}-109=0 .
\end{aligned}
$$

Again we have carried out this process in exact integer arithmetic to emphasize that this equation is an exact representation of the intersection points. Some considerations for floating-point implementation are listed

\begin{tabular}{|c|c|c|c|}
\hline $\begin{array}{l}\text { Intersection } \\
\text { Number }\end{array}$ & $\begin{array}{c}t_{1} \\
\text { Parameter } \\
\text { of Curve } 1\end{array}$ & $\begin{array}{c}\text { Coordinates } \\
\text { of Point }\end{array}$ & $\begin{array}{c}t_{2} \\
\text { Parameter } \\
\text { of Curve } 2\end{array}$ \\
\hline 1 & 0.0621 & $4.2982,2.3787$ & 0.3489 \\
\hline 2 & 0.1098 & $4.4556,2.9718$ & 0.1330 \\
\hline 3 & 0.1785 & $4.6190,3.4127$ & 0.9389 \\
\hline 4 & 0.3997 & $4.9113,3.2894$ & 0.9219 \\
\hline 5 & 0.4212 & $4.9312,3.2186$ & 0.0889 \\
\hline 6 & 0.6828 & $5.1737,2.2902$ & 0.5339 \\
\hline 7 & 0.8610 & $5.4676,2.3212$ & 0.5944 \\
\hline 8 & 0.9342 & $5.6883,2.8773$ & 0.8463 \\
\hline 9 & 0.9823 & $5.9010,3.6148$ & 0.0369 \\
\hline
\end{tabular}
elsewhere.16

We now compute the roots of this degree-nine polynomial. Those roots are the parameter values of the points of intersection. The $x, y$ coordinates of those intersection points can be easily found from the parametric equations of the second curve, and the parameter values on the first curve for the intersection points can be found from the inversion equations. The results are presented in Table 1.

The most common curve intersection algorithms are based on subdivision. Tests indicate that the implicitization algorithm is several times faster than subdivision methods for curves of degree two and degree three, and subdivision methods are faster for curves of degree five and greater. ${ }^{16}$

\section{Summary}

We have presented a tutorial on resultants, curve implicitization, curve inversion, and curve intersection. Interest in this research is growing, and it is evident that algebraic geometry is a valuable resource for computeraided geometric design. 


\section{References}

1. A. Kurosh, Higher Algebra, MIR Publishers, Moscow, 1975.

2. G. Salmon, Modern Higher Algebra, Hodges, Smith, and Co., Dublin, 1866.

3. R.M. Winger, An Introduction to Projective Geometry, D.C. Heath and Co., 1923.

4. A.L. Dixon, "The Eliminant of Three Quantics in Two Independent Variables," Proc. London Math. Soc., Vol. 6, Ser. 2, 1908, pp.468-478.

5. F.S. Macaulay, The Algebraic Theory of Modular Systems, Cambridge University Press, Cambridge, 1916.

6. G.E. Collins, "The Calculation of Multivariate Polynomial Resultants," J. ACM, Vol. 18, No. 4, Apr. 1971, pp.515-532.

7. S.Y. Ku and R.J. Adler, "Computing Polynomial Resultants: Bezout's Determinant vs. Collins' Reduced P.R.S. Algorithm," Comm. ACM, Vol. 12, No. 1, Jan. 1969, pp.23-30.

8. J.E. Rowe, "The Equation of a Rational Plane Curve from Its Parametric Equations (Second Paper)," Bulletin American Math. Soc., Vol. 23, 1917, pp.304-307.

9. G. Salmon, Higher Plane Curves, G.E. Stechert \& Co., New York, 1934

10. T.W. Sederberg, "Implicit and Parametric Curves and Surfaces for Computer-Aided Geometric Design," PhD dissertation, Purdue University, West Lafayette, Ind., 1983.
11. T.W. Sederberg, D.C. Anderson, and R.N. Goldman, "Implicit Representation of Parametric Curves and Surfaces," Computer Vision, Graphics and Image Processing, Vol. 28, 1984, pp.72-84.

12. J. Kajiya, "Ray Tracing Parametric Patches," Computer Graphics, Vol. 16, No. 3, Mar. 1982, pp.245-254.

13. Y. de Montaudouin and W. Tiller, "The Cayley Method in Computer-Aided Geometric Design," Computer Aided Geometric Design, Vol. 1, No. 4, Dec. 1984, pp.309-326.

14. R.N. Goldman, T.W. Sederberg, and D.C. Anderson, "Vector Elimination: A Technique for the Implicitization, Inversion, and Intersection of Planar Parametric Rational Polynomial Curves," Computer Aided Geometric Design, Vol. 1, No. 4 , Dec. 1984, pp.327-356.

15. T.W. Sederberg, R.N. Goldman, and D.C. Anderson, "Implicitization, Inversion and Intersection of Rational Cubic Curves," Computer Vision, Graphics and Image Processing, Vol. 31, 1985, pp.89-102.

16. T.W. Sederberg and S. Parry, "A Comparison of Three Curve Intersection Algorithms," Computer Aided Design (to appear).

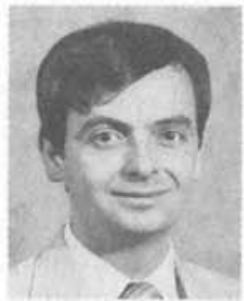

Thomas W. Sederberg is an assistant professor in the Civil Engineering Department at Brigham Young University. His research interests include computer-aided geometric design, free-form curves and surfaces, solid modeling, and computer graphics. He received a BS and an MS in civil engineering from Brigham Young and $\mathrm{ahD}$ in mechani$\mathrm{cal}$ engineering from Purdue University. $\mathrm{He}$ is a member of ACM and IEEE.

Sederberg's address is Brigham Young University, Provo, UT 84602.



Ronald N. Goldman is a principal consultant at Control Data where his interests and responsibilities include a full spectrum of topics in computer-aided geometric design and solid modeling. Since 1977 he has worked in industry, first at MDSI in Ann Arbor, Michigan, where he was the research and development mathematician for the Design solidmodeling project, and later at Ford Motor Company in Dearborn, where he worked on enhancing the in-house design graphics system. He also taught mathematics as an assistant professor, first at the University of Wisconsin, Madison, and later at the University of Michigan, Ann Arbor.

Goldman received a BS in mathematics from MIT in 1968 and an MA and $\mathrm{ahD}$ in mathematics from Johns Hopkins University in 1973.

Goldman's address is Control Data Corp., 4105 N. Lexington Ave., AHS-251, Arden Hills, MN 55126.

\section{The Swiss Federal Institute of Technology at Lausanne, Switzerland \\ invites applications for three newly created professorships in
NUMERICAL ANALYSIS AND SIMULATION \\ APPLIED STATISTICS \\ ARTIFICIAL INTELLIGENCE \\ Applications deadline: June 30th, 1986 \\ Beginning of activity: Fall 1986 or later \\ Further information at the following address:

$$
\begin{gathered}
\text { Secrétariat général de l'Ecole polytechnique, } \\
\text { f'ed'erale de Lausanne, CE-Ecublens, } \\
1015 \text { Lausanne, Suisse }
\end{gathered}
$$

